\title{
Local Communication of Multiple Mobile Robots: Design of Optimal Communication Area for Cooperative Tasks
}

\author{
Eiichi Yoshida* \\ Division of Systems Science \\ Mechanical Engineering Laboratory \\ AIST, MITI \\ Ibaraki 305-8564, Japan \\ e-mail:eiichi@mel.go.jp
}

\section{Tamio Arai, Masakazu Yamamoto, and} Jun Ota

Department of Precision Machinery Engineering School of Engineering

The University of Tokyo

Tokyo 113-8656, Japan

Received April 16, 1997; accepted February 24, 1998

This paper presents an optimal design for local communication between multiple mobile robots. In previous studies of local communication in multirobot systems, the area of communication was not designed using mathematical analysis, but only time-consuming simulations of multirobot communications. We analyzed the information transmission efficiency and created an optimal communication area that minimizes the information transmission time to multiple robots. This optimization comprises two steps. First, we derive the "information transmission probability" for various task models. Next, the derived information transmission probability is used to minimize the information transmission time. The optimal communication design is tested for various tasks, using system parameters. The analytical results are further verified by using computer simulations of multirobot communications and experiments with local communication. (c) 1998 John Wiley \& Sons, Inc.

\footnotetext{
* To whom all correspondence should be addressed.
} 


\section{INTRODUCTION}

Research into mobile robotics has recently made remarkable progress; mobile robots now perform sophisticated and complicated tasks through cooperation. Many studies are being conducted on cooperation, which enables a group of simple robots to accomplish tasks such as exploration, ${ }^{1-7}$ map generation, ${ }^{8,9}$ and transportation. ${ }^{10-13}$

The above cooperation generally requires interrobot communication. Many robotic researchers have studied this subject, and those studies can be classified into global and local inter-robot communication. When only a few robots are included in the cooperative system, global communication, ${ }^{14-16}$ in which the information transmission is centrally controlled, works well. In an environment where there are many robots, however, it has the following drawbacks:

- The information transmission efficiency becomes low when a single communication medium is assigned to many robots, as in a radio network.

- If a central station manages the communication in the area, an increased load may cause a communication bottleneck and insufficient fault tolerance.

- In most cases, a robot can determine its behavior by using local communication only and does not need global information from the whole system.

For these reasons, local communication is used more frequently. ${ }^{7,17,18}$ for many-robot systems. This paper also introduces a local communication model in which the information is sent within a limited area in the form of a packet. This model offers the following advantages:

- The communication can be easily implemented by using an infrared device ${ }^{19}$ or camera image. ${ }^{20}$

- Information transmission takes place in a distributed and concurrent manner, which reduces excessive information processing.

- The overall system becomes robust against the addition, removal, or breakdown of the robots.

The local communication model is therefore suitable for a distributed system with many mobile robots.
Information about a specific task is diffused among the robots through repeated local transmission and robot motion.

This paper deals with cooperative tasks consisting of the following two steps, shown in Figure 1:

(a) Communication regarding a task to the number of robots required by the task. This information would include the attributes of multiple tasks (e.g., the place and type of task).

(b) Communication regarding task execution. This information would include the status of task execution (e.g., constructing a map in the case of a cooperative map generation task).

These procedures can generally be applied to most multirobot cooperative tasks.

Step (a) is important, since it is necessary whenever a cooperative task is executed. The task information only needs to be transmitted locally to the required number of robots. In step (b) also, tasks like map generation or cooperative transfer can be executed through a local information exchange between the robots involved.

Thus cooperative tasks that consist of steps (a) and (b) can be realized by using the simple local communication model. The same information packet

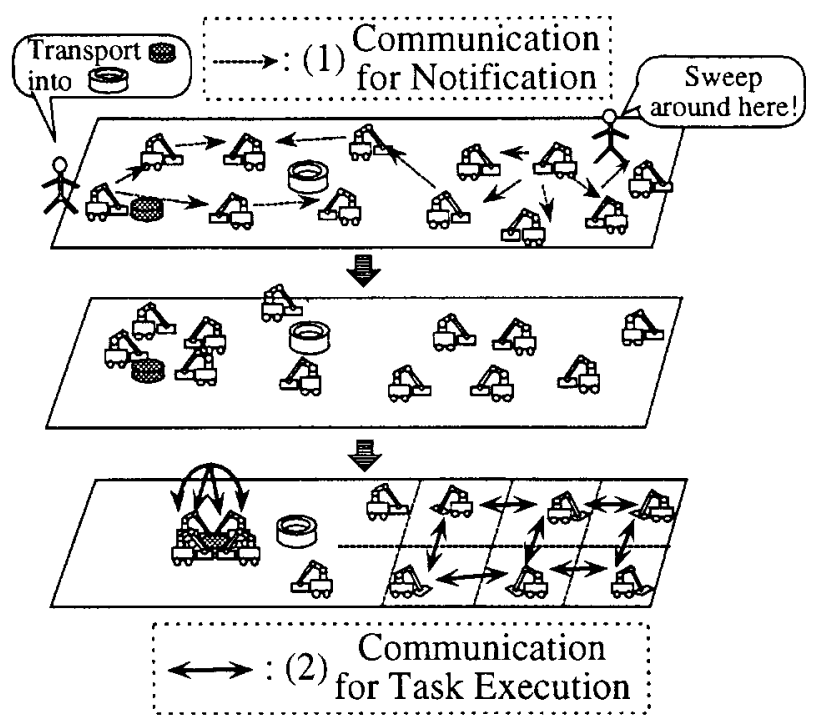

Figure 1. Two types of communication for cooperation. 
is sent out several times to ensure reliability due to stochastic transmission characteristics (as with a computer network).

Task execution performance is improved by reducing the time required for transmitting the task information from the task detector to nearby robots in communication (a), and among cooperating robots in (b). The communication area design is very important because it takes too long for information to be received by the required robots if the local communication area is too large or too small.

Most recent research utilizes local communication for multiple mobile robot systems. ${ }^{7,17,18,21}$ The communication area, however, has been determined through trial and error from many simulation trials. This time-consuming design procedure using simulations must be repeated every time changes are made in the robotic system. Although the communication area design is important, using a mathematical analysis of the information transmission is rarely discussed.

There are some studies in the field of communication theory, ${ }^{22}$ but they involve radio computer network systems and are not directly applicable in dynamic systems in which various cooperative tasks are given and robots are moving continuously.

This paper aims to establish a design method for an optimal communication area based on analysis. As a preliminary step, we model several cooperative tasks and information transmission using stochastic distributions and a differential equation. The optimal communication area is derived by minimizing the average transmission time, which is used as the evaluation function in this paper. The effectiveness of the design method is verified by computer simulations and experiments using multiple robots.

\section{LOCAL COMMUNICATION MODEL}

\subsection{Local Communication Model}

We employed a simplified model, briefly described below.

(1) Each robot sends out information in the form of a packet within limited area $A$.

(2) Each robot executes an information reception process following each time unit long enough for information acquisition. If there is any available information, the robot receives it.
(3) Each robot sends out an information packet with probability $p_{e}$ within a time unit.

(4) There is an upper limit $c$ in the number of robots from which a robot can obtain packets.

(5) All robots have the same communication and motion capacities.

The capacity of the information packet in (1) is determined so that it can include the contents explained in the previous section. Activity (1) also simulates a local broadcast model, as illustrated in Figure 2.

Based on the content of information $\mathscr{I}$, we define the robots that have already obtained $\mathscr{I}$ as I-Robots (Informed), and those not informed as $\mathrm{N}$ Robots (Not Informed).

In (2) above, the time is regularized in such a way that the analysis is generally applicable to various cases. Parameter $p_{e}$ in (3) represents how frequently each robot transmits an information packet during task execution (Fig. 2). If sparsely scattered robots are conducting an exploring task in a large area, they can spend most of the time for the task and do not need to communicate so often. By contrast, transporting a heavy object by many robots may require frequent information exchange to correct position errors. The interval $T_{\text {com }}$ between information transmission can be determined according to the cooperative task in this way. If $T_{c o m}$ is longer than a time unit, $p_{e}$ equals to the reciprocal of $T_{\text {com }}$; otherwise, $p_{e}$ equals $T_{c o m}$. Value $c$ in (4) corresponds to the communication capacity of the robots. We call $p_{e}$ the information output probability and $c$ the information acquisition capacity. If a robot finds more than $c$ robots transmitting information, we propose two possibilities:

- The robot cannot receive any information packets (interfering communication);

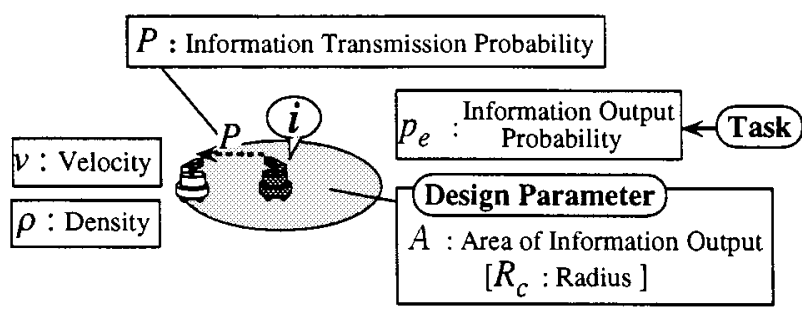

$x(=\rho A):$ Average Number of Robots in Output Area

Figure 2. Model of local communication. 


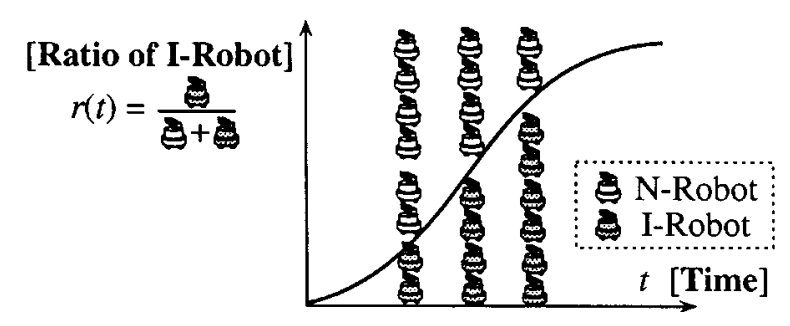

Figure 3. Information diffusion among robots.

- The robot receives information packets from $c$ robots (noninterfering communication).

An example of the former case is infrared or radio communication, and the latter corresponds to communication using images.

We define information transmission probability $P$ as the probability that a robot can successfully receive information sent out by another robot. This measure is important when the communication area is optimized in the analysis that follows. During communication steps (a) and (b) defined in Section 1 , information is diffused between robots by repeated local transmissions and robot motion. We refer to the ratio of I-Robots for the specific content of information $\mathscr{I}$ at time $t$ as the information diffusion ratio $r(t)$, which represents the information diffusion process shown in Figure 3.

The design parameter in this paper is communication area $A$ or radius $R_{c}$. As explained later, the input parameters of the design are the robot density $\rho$ in the environment, and $p_{e}$ and $c$ (determined from tasks and the robots' capacity). Value $x$, shown in Figure 2, is defined as the product of $\rho$ and $A$ and denotes the average number in the communication area. In the course of the analysis in Section 3, we use $x$ as the design parameter to give a clearer perspective.

\subsection{Models of Cooperative Tasks}

We modeled three typical cooperative tasks (Fig. 4) for multiple robots:

(i) Random search of the area. ${ }^{2,8}$ Robots move randomly searching the environment.

(ii) Cooperative transfer. ${ }^{11-13}$ Robots cooperatively transport a circular object and are disposed on the circumference (Fig. 4(ii)).

(iii) Search in assigned area., ${ }^{4,5}$ Each robot searches an assigned square area (Fig. 4(iii)).

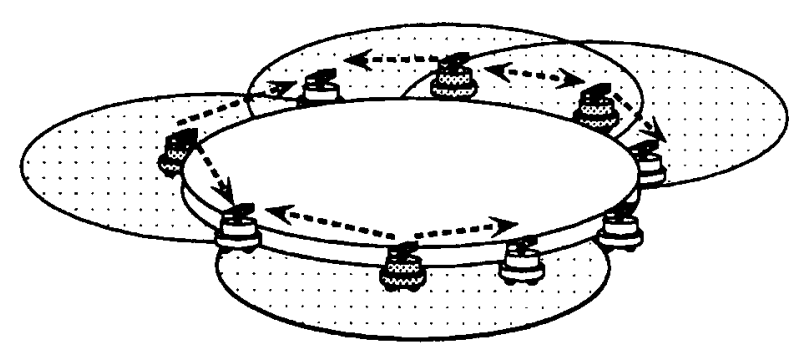

(ii)

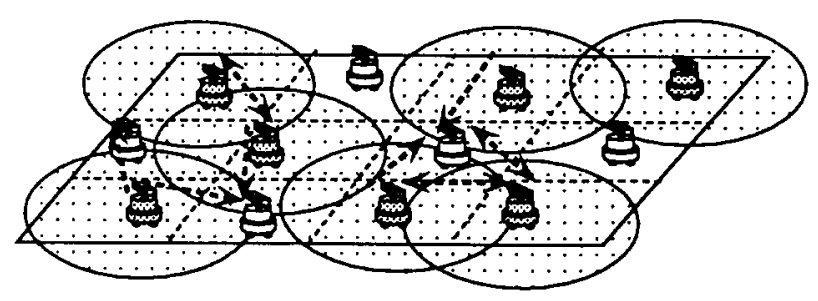

(iii)

Figure 4. Model of cooperative tasks. (ii) Cooperative transfer. (iii) Search in assigned area.

Task (i) is a fundamental search and is mainly used as the task notification (a). In (ii) and (iii), the radius of the circle and the length of the edge are normalized to a unit length so that the analysis can be extended to other dimensions. Robots are located randomly on the circumference or in the square area to accommodate various task configurations.

The motion of the robot is determined by the task and the robot mechanism. We define $v$ as the velocity of motion and $\mathscr{M}$ as the set of other parameters that determine the robots' movements.

From these contents given in Section 2.1, the information diffused between robots can be described by

(a) Notification: information about multiple tasks;

(b) task execution: parts changed by robots in a common data format (such as a map) utilized in the execution of the task.

Since the packet can include information regarding multiple tasks and the changed parts of the data format, the different information contents are diffused independently.

Table I summarizes the design and input parameters. The evaluation function to be mini- 
Table I. Parameters of local communication system.

\begin{tabular}{|c|c|c|c|}
\hline $\begin{array}{l}\text { Types of } \\
\text { parameters }\end{array}$ & Symbol & Variable & $\begin{array}{l}\text { Critical factors } \\
\text { deciding variable }\end{array}$ \\
\hline $\begin{array}{l}\text { Design } \\
\text { parameters }\end{array}$ & $\begin{array}{l}A \\
R_{c}\end{array}$ & Communication area & \\
\hline $\begin{array}{l}\text { Input } \\
\text { Parameters }\end{array}$ & $\begin{array}{l}\rho \\
v \\
\mathscr{M} \\
c \\
p_{e}\end{array}$ & $\begin{array}{l}\text { Robot density } \\
\text { Velocity of motion } \\
\text { Type of motion } \\
\text { Information acquisition capacity } \\
\text { Information output probability }\end{array}$ & $\begin{array}{l}\text { Constant in the environment } \\
\text { Robot mechanism } \\
\text { and task specs } \\
\text { Communication capacity } \\
\text { Task specs }\end{array}$ \\
\hline
\end{tabular}

mized is the transmission time $W$, as mentioned in Section 1.

\section{FORMULATING THE INFORMATION TRANSMISSION}

The goal of this paper is to derive the communication area $A_{\text {opt }}$ (or $R_{\text {copt }}$ ), which minimizes the information transmission time $W$ (the evaluation function). Since $W$ can be represented by using the information transmission probability $P, A_{\text {opt }}\left(R_{\text {copt }}\right)$ is obtained by the following steps (A) and (B), also outlined in Figure 5:

(A) $P$ is computed using the parameters defined in Section 2.

(B) $x_{o p t}\left(R_{\text {copt }}\right)$ is derived by minimizing $W$, represented with respect to $P$ (Section 4 ).

This section deals with the analysis corresponding to step (A) in Figure 5. In Section 3.1, the spatial distribution for each task will be modeled, followed by the analysis of $P$. We derive $P$ as $P\left(c, p_{e}, x\right)$ for transmission to an arbitrary robot as the primary case of fundamental communication. Here $x(=\rho A)$ is used in place of $A$ to simplify the analysis. When applying this analysis to a transmission to multiple robots, the information diffusion ratio $r(t)$ must be considered. Consequently, $P$ can be obtained as $P\left(c, p_{e}, x, t\right)$. We refer to $P_{I}$ and $P_{N}$ as $P$ for interfering and noninterfering communication.

\subsection{Modeling Spatial Distribution of Robots}

We model the spatial distribution of the robots for each task explained in Section 2.2 by calculating the following probability:

$\operatorname{Prob}[i \mid i \subset \mathscr{S}(A)] \equiv \operatorname{Prob}[i$ robots exist in the area

(average number in it: $x$ )]

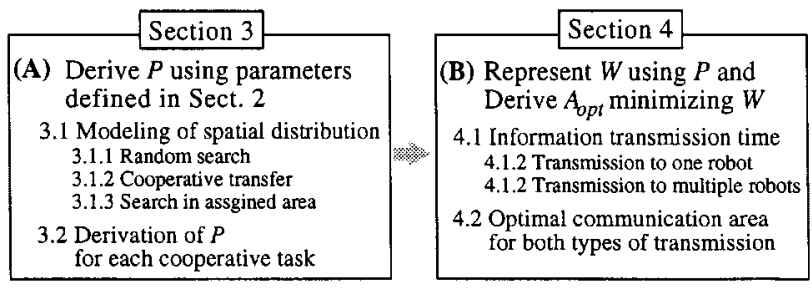

Figure 5. Structure of the analysis.

where $\mathscr{S}(A)$ denotes the region included in communication area $A . \mathscr{S}\left(R_{c}\right)$ in Section 3.1.2 is defined for the cooperative transfer task in the same way.

\subsubsection{Random Search of the Environment}

In the random search task, we assume that each robot changes its moving direction randomly in a range $\pm \theta(0 \leq \theta \leq \pi / 4)$ every $\tau$ unit times.

When points are disposed randomly on a plane, the number of points in a certain area is Poissondistributed. ${ }^{22}$ This characteristic can also be applied when robots individually make random movements, which can be verified by computer simulations. ${ }^{23}$ Then the distribution of robots is expressed as

$$
\operatorname{Prob}[i \mid i \subset \mathscr{S}(A)]=\frac{\{\rho A\}^{i}}{i !} e^{-\rho A}
$$

\subsubsection{Cooperative Transfer}

We assume in this task that robots transfer a circular object by changing their grasping point. ${ }^{11,13}$ The communication range $R_{c}$ is designed for $m$ robots located on the circumference, as shown in Figure 4 (ii). The density of robots around a robot is expressed by the linear density $(m-1) / 2 \pi$. The average number of robots $\mu$ in the communication area 
with a radius of $R_{c}$ is expressed as a product of the density $(m-1) / 2 \pi$ and the length $4 \psi$ of the arc included in the area (Fig. 6). It follows that Prob[ $i \mid i$ $\subset \mathscr{S}(x)$ ] can be expressed using a binomial distribution with a probability $\mu /(m-1)$ that robots exist in the communication area:

$$
\begin{aligned}
\mu & =\frac{2(m-1)}{\pi} \sin ^{-1} \frac{R_{c}}{2} \\
\operatorname{Prob}\left[i \mid i \subset \mathscr{S}\left(R_{c}\right)\right]= & { }_{m-1} C_{i}\left(\frac{\mu}{m-1}\right)^{i} \\
& \times\left(1-\frac{\mu}{m-1}\right)^{m-1-i}
\end{aligned}
$$

\subsubsection{Search in Assigned Area}

The task shown in Figure 4 (iii) assumes that robots search or sweep in certain assigned areas. ${ }^{5}$ The robot density is 1 , since one robot is assigned in a square with area 1.

The spatial distribution of robots in this task varies, depending on communication area $A$. It is difficult to obtain an analytical model applicable for all of $A$; thus we approximate the spatial distribution Prob $[i \mid i \subset \mathscr{S}(A)]$ by using a normal distribution with an average number of robots $\mu$ existing in the communication area and its variance $V$, which are given by computer simulations.

We adopt the following approximation as one model that fits the simulations well:

$$
\mu=\sqrt[3]{(\rho A)^{3}+1}-1, \quad V=0.6 \sqrt{\mu}
$$

\subsection{Information Transmission Probability}

We are now ready to investigate the information transmission probability $P$ by using the spatial distributions derived so far. In the next section, the

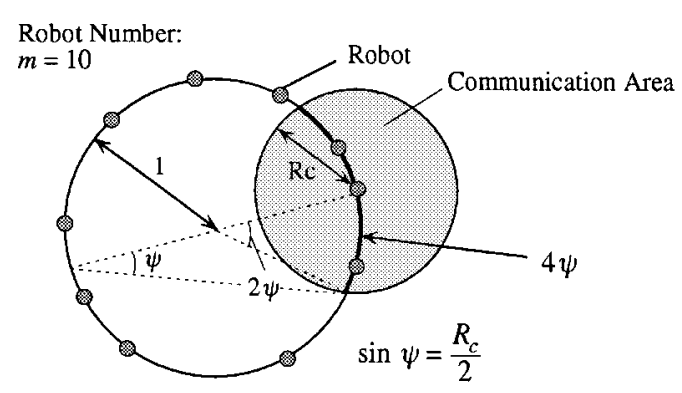

Figure 6. Analysis of spatial distribution in cooperative transfer. information transmission time $W$ is evaluated using $P$.

In the most fundamental case, $P$ is derived as $P\left(c, p_{e}, x\right)$ for transmission to an arbitrary robot. To simplify the analysis, we use $x(=\rho A)$ instead of $A$ for tasks (i) and (iii) in Figure 4. For task (ii), $R_{c}$ is used as the parameter corresponding to $x$; once the fundamental analysis of the simple transmission is finished, it is extended to obtain $P$ for the transmission to multiple robots through information diffusion. We distinguish $P_{\mathrm{I}}$ and $P_{\mathrm{N}}$ for interfering and noninterfering communication in the following analysis.

\subsubsection{Information Transmission Probability to an Arbitrary Robot}

We define the probability $Q_{i j}$ that there are $i$ robots in the communication area and that $j$ out of $i$ robots are sending out information packets. $Q_{i j}$ is the product of $\operatorname{Prob}[i \mid i \subset \mathscr{S}(x)]$ and the binomial distribution with information output probability $p_{e}$. It is then expressed as $Q_{i j}\left(p_{e}, x\right)$ :

$$
Q_{i j}\left(p_{e}, x\right)=\operatorname{Prob}[i \mid i \subset \mathscr{S}(A)]_{i} C_{j} p_{e}^{j}\left(1-p_{e}\right)^{i-j}
$$

Now we consider $P-$ the probability $P$ that a robot can obtain the information sent out from other robots using the derived $Q_{i j}$. The probabilities of the following two independent events should be combined for interfering communication:

- $i \leq c$ : Information is always receivable over the range $j \leq i \leq c$, since at most there are $c$ robots sending out information and no interference occurs.

- $i \geq c+1$ : Information cannot be received unless $j \leq c$ robots out of $i$ send out information. The corresponding probability is given by the summation of $Q_{i j}\left(p_{e}, x\right)$ for $i \geq c+1$, $j \leq c$.

The $P_{\mathrm{I}}$ in (6) is obtained by adding the two terms. For interfering communication, $c$ robots can obtain information even if there are more than $c$ sending robots. This is calculated by

- $j \geq c+1$ : Information can be received from $c$ out of $j$ robots sending information. The probability of reception of a specific content of information is $(c / j) Q_{i j}\left(p_{e}, x\right)$. The corresponding term is calculated as the summation of $(c / j) Q_{i j}\left(p_{e}, x\right)$ over the range $i \geq c+1$, $i \geq j \geq c+1$. 
In (7) below, $P_{\mathrm{N}}$ is obtained by adding this term to $P_{\mathrm{I}}$ :

$$
\begin{aligned}
P_{I}\left(c, p_{e}, x\right)= & \sum_{i=1}^{c} \sum_{j=1}^{i} Q_{i j}\left(p_{e}, x\right) \\
& +\sum_{i=c+1}^{\infty} \sum_{j=1}^{c} Q_{i j}\left(p_{e}, x\right) \\
P_{N}\left(c, p_{e}, x\right)= & P_{I}\left(c, p_{e}, x\right) \\
& +\sum_{i=c+1}^{\infty} \sum_{j=c+1}^{i} \frac{c}{j} Q_{i j}\left(p_{e}, x\right)
\end{aligned}
$$

\subsubsection{Information Transmission Probability to Multiple Robots}

In this case, $P$ takes different values according to the diffusion ratio of information. The information diffusion ratio $r(t)$ represents the ratio of an I-Robot with respect to a specific content of information $\mathscr{I}$ at time $t$. The following analysis considers whether information $\mathscr{I}$ is included when a robot receives a packet.

We defined $Q_{i j}\left(p_{e}, x\right)$ as the probability that there are $j$ sending robots among $i$ of those in the communication area. Let $\bar{r}_{j}(t)$ be the probability that there is at least one I-Robot for $i$ among $j$ robots. This is given as

$$
\bar{r}_{j}(t)=1-(1-r(t))^{j}
$$

Thus $P\left(c, p_{e}, x, t\right)$, with respect to information $\mathscr{I}$ at time $t$, is computed for interfering and noninterfering communication from (6) and (7) as follows:

$$
\begin{aligned}
P_{I}\left(c, p_{e}, x, t\right)= & \sum_{i=1}^{c} \sum_{j=1}^{i} Q_{i j}\left(p_{e}, x\right) \bar{r}_{j}(t) \\
& +\sum_{i=c+1}^{\infty} \sum_{j=1}^{c} Q_{i j}\left(p_{e}, x\right) \bar{r}_{j}(t) \\
P_{N}\left(c, p_{e}, x, t\right)= & P_{I}\left(c, p_{e}, x, t\right) \\
& +\sum_{i=c+1}^{\infty} \sum_{j=c+1}^{i} Q_{i j}\left(p_{e}, x\right) \bar{r}_{c}(t)
\end{aligned}
$$

\section{OPTIMAL COMMUNICATION AREA}

Step (B) in Figure 5 is described in this section. After analyzing the information transmission time $W$ as the evaluation function, we will derive the optimal communication area that minimizes $W$ for transmission to an arbitrary robot and to multiple robots. The analysis below deals mainly with interfering communication, since the same computation applies for noninterfering communication.

As mentioned in Section 3.2, we use the average number of robots $x(=\rho A)$ instead of communication area $A$ to clarify the analysis in tasks (i) and (iii). Since $\rho$ is a constant value in the environment, $A_{\text {opt }}$ is easily calculated from the optimal value $x_{\text {opt }}$ as $x_{\text {opt }} / \rho$. It is also straightforward to know the characteristics of $A$, since $x$ is proportional to $A$.

In the following analysis, we will derive the $x_{\text {opt }}\left(R_{\text {copt }}\right.$ in task (ii)) that minimizes $W$.

\subsection{Information Transmission Time}

\subsubsection{To an Arbitrary Robot}

In this case, the information transmission time $W$ is the number of time units elapsed before the output information is transmitted to another arbitrary robot. Since the probability that the information is transmitted at each time unit is $P$, the probability that it takes $k$ time units to succeed in transmission is expressed as the geometric distribution $P(1-P)^{k-1}$. The average transmission time $W$ is calculated by

$$
W=\frac{1}{P}
$$

as seen in the literature. ${ }^{24}$ The value of $x_{o p t}$ that minimizes $W$ is obtained by maximizing $P$ in (6) and (7) for transmission to an arbitrary robot.

\subsubsection{To Multiple Robots}

To calculate the $x_{o p t}$ that minimizes the transmission time to multiple robots, the information diffusion process is analyzed, taking the robots' motion into consideration. The differential equation for the information diffusion ratio $r(t)$ is derived based on our previous analysis. ${ }^{23}$ The increment of $r(t)$ per time $\Delta t, \Delta r(t)$, corresponds to the ratio of newly generated I-Robots at time $t$ and is proportional to the product of $P$ and the ratio of N-Robots $1-r(t)$. We can express the equation of information diffusion using $r(t)$ and $P\left(c, p_{e}, x, t\right)$ in (9) or (10) as

$$
\frac{d r(t)}{d t}=\beta(v, \mathscr{M}, x) P\left(c, p_{e}, x, t\right)\{1-r(t)\}
$$

In the equation, $\beta(v, \mathscr{M}, x)$ is a coefficient representing the effect of robot motion parameters such as $v$ and $\mathscr{M}$.

On the left side of Eq. (12), $d r(t) / d t$ represents the "diffusion velocity"; the larger the value of 
$d r(t) / d t$, the more rapidly information is diffused. We can obtain $x_{\text {opt }}$, minimizing $W$ to multiple robots, by calculating $x$, which maximizes $\beta(v$, $\mathscr{M}, x) P\left(c, p_{e}, x, t\right)$ in the right side of Eq. (12).

\subsection{Optimal Communication Area}

\subsubsection{To An Arbitrary Robot}

To compute $x_{o p t}$, first we express $P$ as the function of $\left(p_{e}, x\right)$ for the fixed information acquisition capacity $c$. The graph of $P$ for constant $c$ looks like Figure 7 , in terms of $\left(p_{e}, x\right)$. The example in Figure 7 concerns the case of a random search task, $c=1$, interfering communication.

Next, according to information output probability $p_{e}$, which varies depending on the content of the task, the optimal communication are $x_{o p t}$ is obtained as the value of $x$ maximizing $P$, indicated by Max of $P_{\mathrm{I}}$ in Figure 7.

The values of $x_{o p t}$ are plotted in terms of $p_{e}$ in Figure 8 , which is the projection onto a plain $\left(p_{e}, x\right)$ of the curve represented by Max of $P_{\mathrm{I}}$ in Figure 7. We can easily obtain the values of $x_{o p t}$ for various $p_{e}$ from this graph.

For a random search task with interfering communication, $P_{\mathrm{I}}\left(c, p_{e}, x\right)$ is arranged from (2) and (6) as follows:

$$
P_{I}\left(c, p_{e}, x\right)=e^{-p_{e} x}\left(\sum_{k=0}^{c} \frac{\left(p_{e} x\right)^{k}}{k !}-1\right)
$$

By solving $(d / d x) P\left(c, p_{e}, x\right)=0$, we can derive the

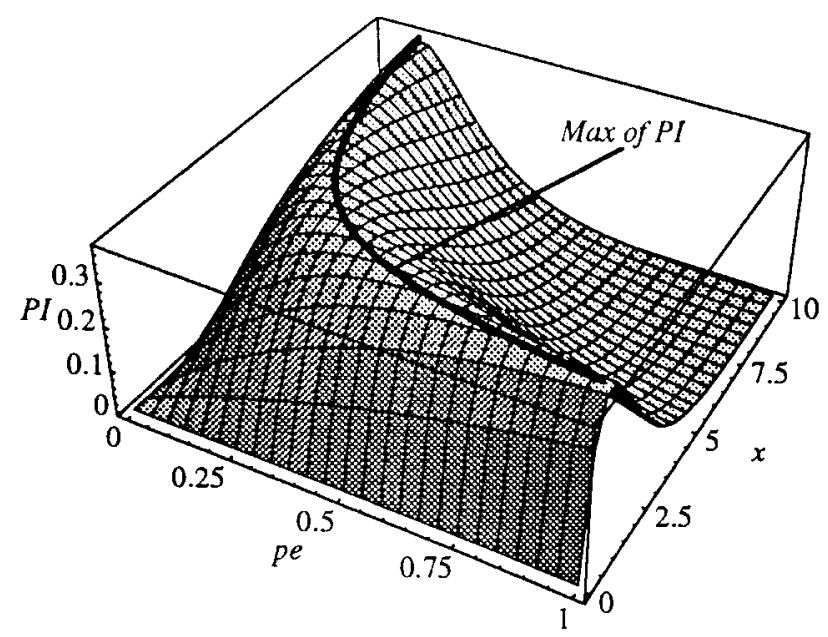

Figure 7. $P$ plotted versus $\left(p_{e}, x\right)($ random search, $c=1)$.

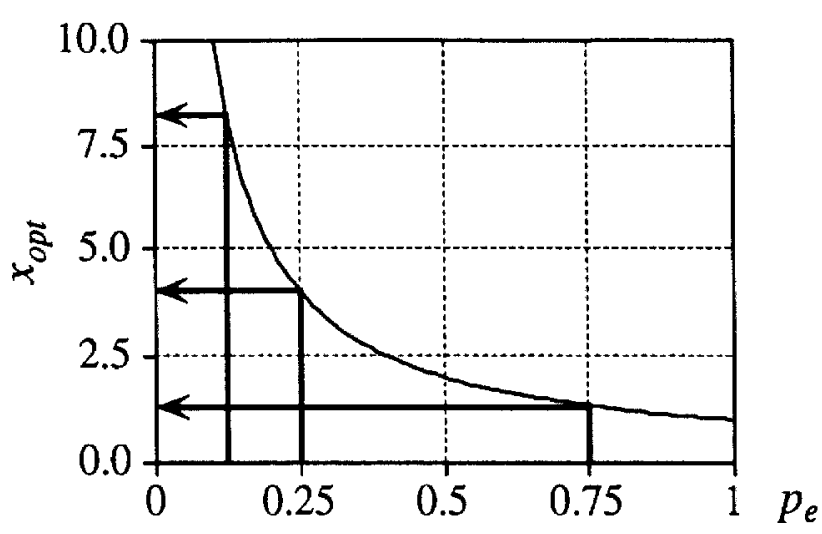

Figure 8. $x_{\text {opt }}$ plotted versus $p_{e}($ random search, $c=1)$.

$x_{\text {opt }}$ that maximizes $P$ by a simple formula:

$$
x_{o p t}=\sqrt[c]{\frac{c !}{p_{e}^{c}}}=\frac{\sqrt[c]{c !}}{p_{e}}
$$

In (14), $x_{o p t}$ is in inverse proportion to the information output probability $p_{e}$. This indicates that the communication area should be small (large) when the frequency of information transmission is high (low), which agrees with our findings.

The result in (14) also implies that $x_{o p t}$ increases as the information acquisition capacity $c$ becomes larger. Therefore, the communication range can be enlarged for improved communication capacity. However, we must note that $x_{o p t}$ is not simply proportional to $c$, as in $x_{o p t}=c / p_{e}$.

We can also estimate the optimal radius $R_{\text {copt }}$ of the communication area for the cooperative transfer task. The probability $P_{\mathrm{I}}$, corresponding to the graph in Figure 7, is derived using the total robot number $m$ and the information acquisition capacity c. It is given as follows, using $\mu$ :

$$
P_{I}\left(c, p_{e}, \mu\right)=\sum_{i=1}^{c}{ }_{m-1} C_{i}\left(\frac{\mu p_{e}}{m-1}\right)^{i}\left(1-\frac{\mu p_{e}}{m-1}\right)^{m-1-i}
$$

Similar to the random search task, $R_{\text {copt }}$ is obtained by calculating the $\mu_{\text {opt }}$ that maximizes $P_{\mathrm{I}}$ in accordance with a given $p_{e}$. This is sufficient to derive the graph in Figure 8, and $\mu_{o p t}$ is inversely proportional to $p_{e}$.

We can also model $x_{o p t}$ for the task of searching in an assigned area. Furthermore, the optimization method is also applicable to noninterfering communication. 


\subsubsection{To Multiple Robots}

Applying the same analytical procedure to transmission to an arbitrary robot, we derive the optimal communication area with which the part $\beta(v, \mathscr{M}$, $x) P\left(c, p_{e}, x, t\right)$ in (12) takes the minimum as the evaluation function.

The coefficient $\beta$, which represents the effect of robot motion on information diffusion, is determined by the velocity $v$, the period, and the range of motion direction change $\tau, \theta{ }^{23}$ When information is transmitted to multiple robots, $x_{o p t}$ can be modeled as a function inversely proportional to $p_{e}$, as in $x_{o p t}=f(c) / p_{e}+g(c)$. For instance, $x_{\text {opt }}$ is calculated as 0.85 for the random search task with interfering communication, whose principal parameters are $c=$ 1 and $p_{e}=1.0$. Using formula (13), the equation of information diffusion (12) becomes

$$
\begin{aligned}
& \frac{d r(t)}{d t}=\operatorname{ar}(t)\{1-r(t)\} \\
& \text { where } a=\beta(v, \mathscr{M}, x) e^{-p_{e} x} p_{e} x
\end{aligned}
$$

This is a logistic equation whose solution $r(t)$ is

$$
r(t)=\frac{1}{1+((1-r(0)) / r(0)) e^{-a t}}
$$

where $r(0)$ is the initial value of $r(t)$ at $t=0$. The information diffusion process $r(t)$ shown in (17) is plotted in terms of time $t$ in Figure 9 with robot density $\rho=0.125$, visible angle $360^{\circ}$, and motion parameters $(v, \theta, \tau)=\left(0.2,60^{\circ}, 3\right.$ [time units]).

It is observed that information is transmitted most rapidly with an optimal area of $x_{o p t}=0.85$, while the diffusion is slower if $x$ is larger or smaller than $x_{o p t}$. This demonstrates that the designed optimal communication area achieves the minimum transmission time.

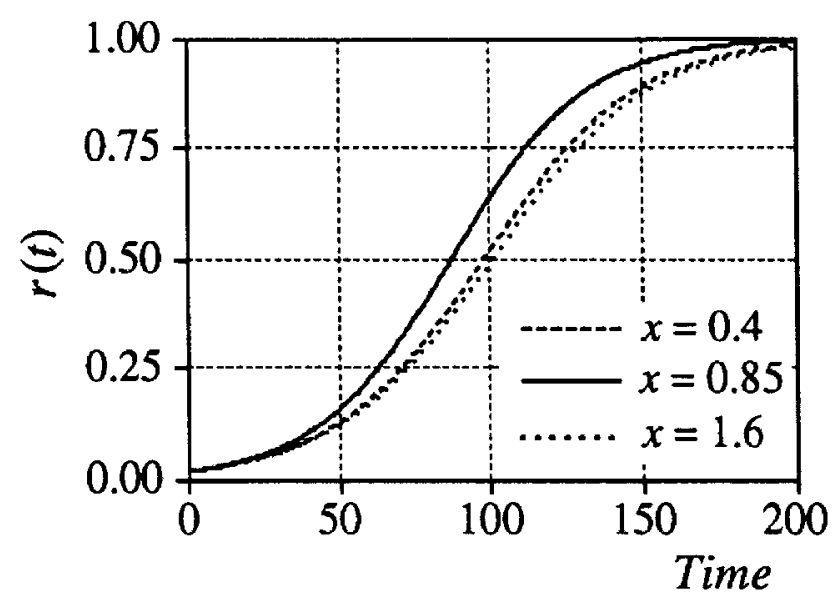

Figure 9. Comparison of information diffusion $r(t)(c=$ $\left.1, p_{e}=1.0\right)$.

\section{INFORMATION TRANSMISSION SIMULATION}

We conducted several simulations to verify the analysis of information transmission and optimal communication area. Local communication between multiple cooperating robots is implemented on computers in the simulations. This chapter discusses only the interfering communication computed in (6) and (9), since noninterfering communication can also be verified by considering the additional terms in (7) and (10).

\subsection{To An Arbitrary Robot}

Multiple mobile robots that perform the tasks modeled in Section 2.2 were implemented on computers using $\mathrm{C}++$ programming language. The information is simulated for various $p_{e}$ in each task, using the parameters shown in Table II.

Table II. Simulation environments for various tasks.

\begin{tabular}{lccc}
\hline & $\begin{array}{c}\text { (i) Random } \\
\text { search }\end{array}$ & $\begin{array}{c}\text { (ii) Cooperative } \\
\text { transfer }\end{array}$ & $\begin{array}{c}\text { (iii) Search in } \\
\text { assigned areas }\end{array}$ \\
\hline Task environment & $5 \times 5$ & $\begin{array}{c}\text { Located randomly } \\
\text { on the circumference }\end{array}$ & $\begin{array}{c}5 \times 5 \\
\text { search area: } \\
1 \times 1 \text { square }\end{array}$ \\
$\begin{array}{l}\text { No. of robots } \\
\begin{array}{l}\text { Design parameter } \\
\text { : Information acquisition } \\
\text { capacity }\end{array}\end{array}$ & 25 & 10 & 25 \\
$p_{e}:$ Information output \\
probability
\end{tabular}




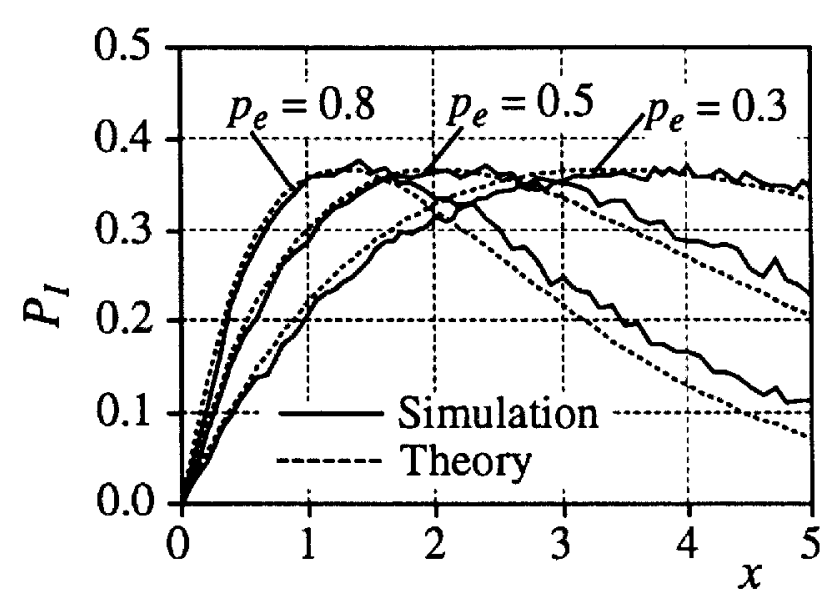

(i)

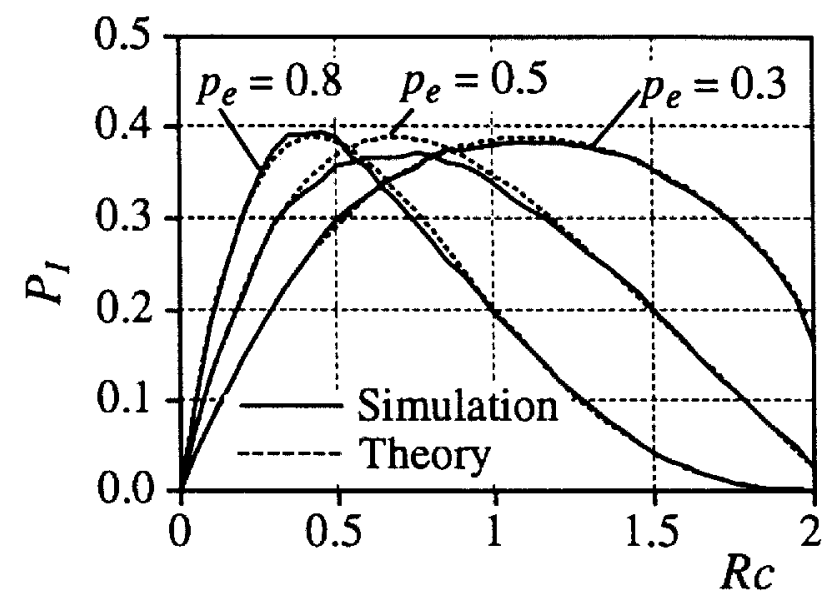

(ii)

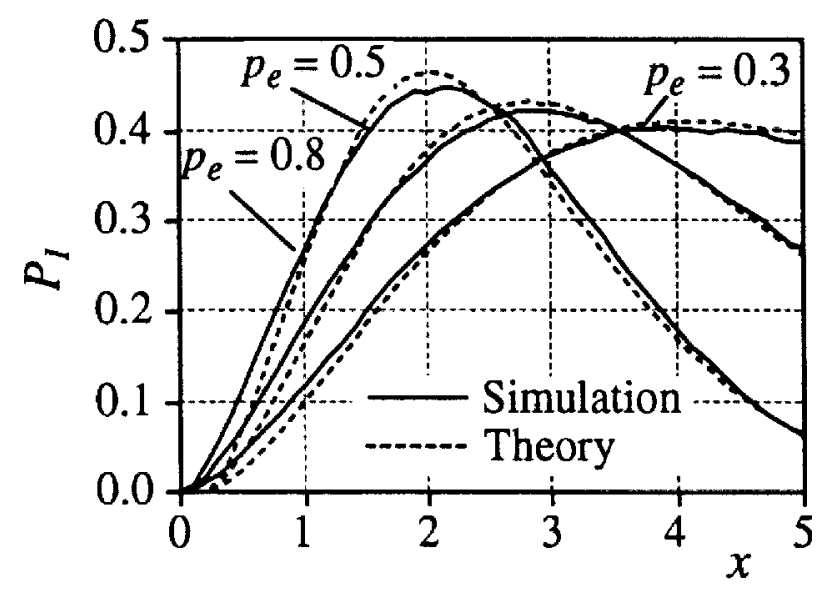

(iii)

Figure 10. Information transmission probability $P_{I}$ versus $p_{e}$. (i) Random search. (ii) Cooperative transfer. (iii). Search in assigned area.
The graphs in Figure 10 (i)-(iii) show the simulation results of $P_{\mathrm{I}}$ (simulation) with the theoretically calculated values (theory) for various communication areas.

The values of the analysis agree well with those of the simulations, and $P_{\mathrm{I}}$ takes the maximum values at the optimal communication areas predicted by the analysis of each task; the effectiveness of the design (based on the analysis) is verified.

In Figure 10 (i), the error becomes significant for a larger value of $x$. This is because in the simulation of a random search, occurrence of interference is less probable when the robots reach the edge of the environment. However, the model is sufficiently accurate near the designed optimal communication area $x_{\text {opt }}$.

\subsection{To Multiple Robots}

Since the analysis of information diffusion was verified as effective in our previous research, ${ }^{23}$ we will only discuss here the validity of the optimal communication area.

Figure 11 shows the simulation results of the information diffusion for a random search task with the same parameters as those used in Figure 9, for $x=0.4,0.85$, and 1.6 in the environment $20 \times 20$ where 50 robots are working.

The values obtained from the analysis correspond well to those of the simulations, and the greatest velocity of information diffusion is observed at the derived optimal communication area $x_{\text {opt }}=0.85$.

The effectiveness of the analysis can be similarly verified for noninterfering communication and

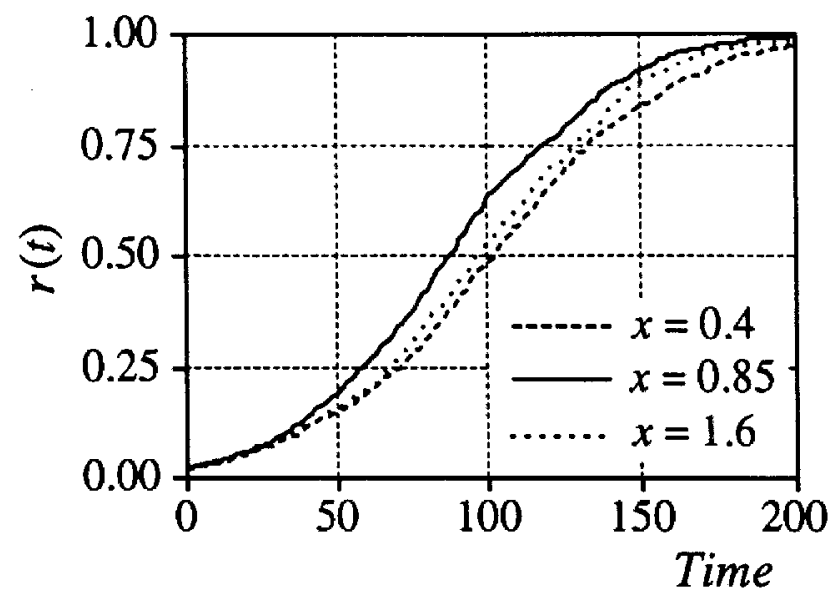

Figure 11. Simulation result of information diffusion $\left(c=1, p_{e}=1.0\right)$. 
other cooperative tasks. The optimal communication area obtained from the analysis is also effective for transmission to multiple robots.

We confirmed that the optimal communication areas we derived are effective in simulations of a robotic environment. We can now optimize the local communication area for multiple mobile robots without time-consuming simulations of information transmission between many robots.

\section{EXPERIMENT WITH INFORMATION TRANSMISSION}

We performed simple experiments on information transmission to verify the design principles of the communication area. Local communication is realized using an infrared device, so that the communication area can be limited and adjusted to the desired distance, as illustrated in Figure 12.

\subsection{System Configuration}

The configuration of infrared local communication system is shown in Figure 13. We used infrared LEDs and sensors in the form of TV remote controls. The modulation was Frequency Shift Keying (FSK), and the transmission rate was 2400 bps. The communication distance could be adjusted from 1 to 4 $\mathrm{m}$ by changing the current to LED from PC via the D/A board.

The infrared LEDs were arranged circularly with the four sensors in the center fro a $360^{\circ}$ communica-

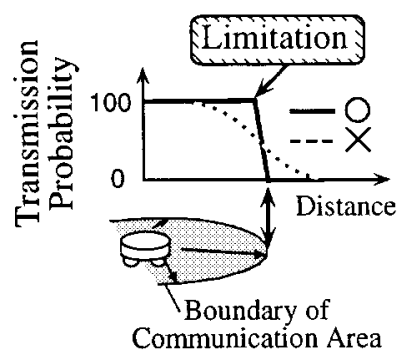

(a)

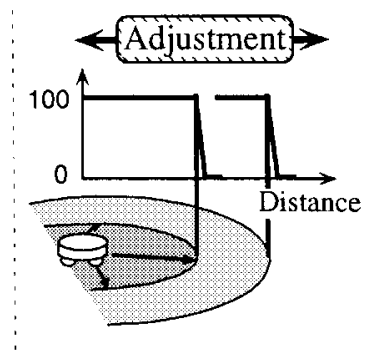

(b)
Figure 12. Limitation and adjustment of local communication area. (a) Limitation. (b) Adjustment.

tion range. Figure 14 shows the communication device mounted on a mobile robot, equipped with a relative position/orientation sensing system using signboards with LEDs and a video camera. ${ }^{20}$

\subsection{Design Verification}

We chose a random search as the basic task and measured the information transmission probability $P$ of the receiver robot from transmitter robots randomly distributed within a 2.5-m distance, as shown in Figure 15.

This was interfering communication, with a robot density $\rho=0.092$, and an information output probability of $p_{e}=1.0$.

The transmitter robots send out 200 bytes of data, changing the communication distance from 1.0 to $2.5 \mathrm{~m}$.

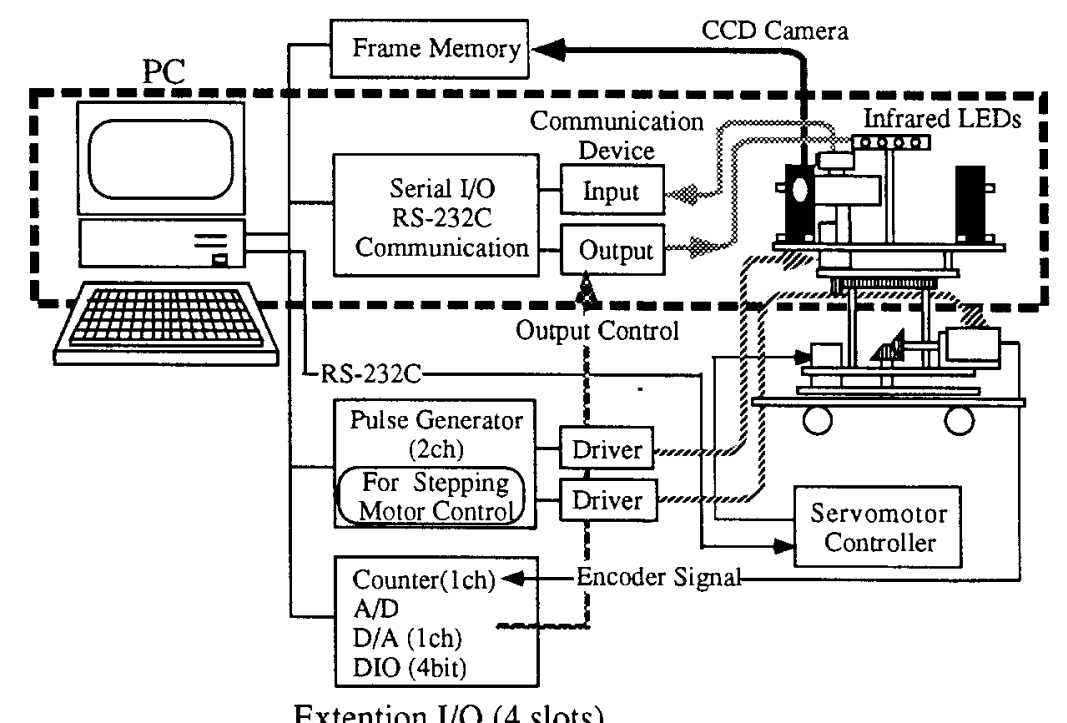

Figure 13. System configuration of infrared local communication system. 


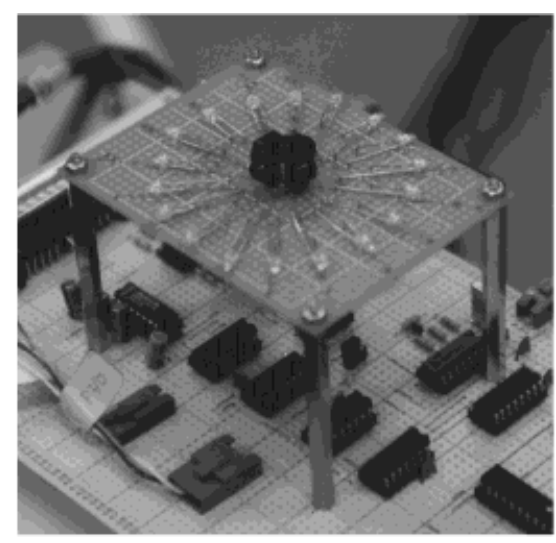

(a)

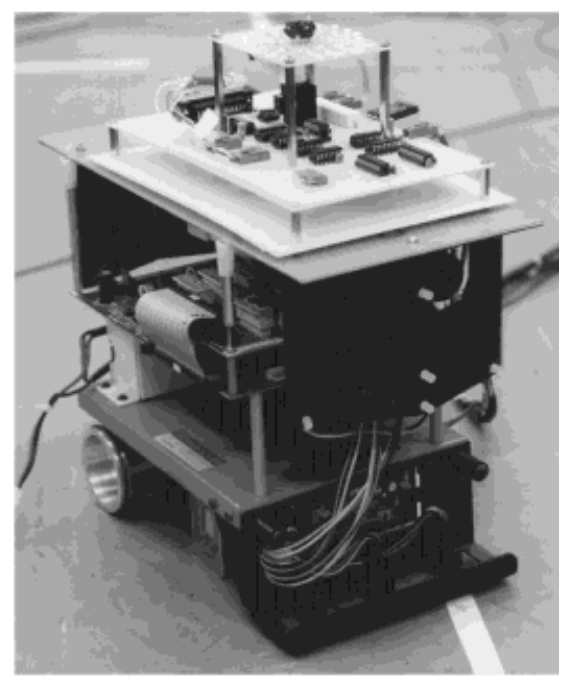

(b)

Figure 14. (a) Infrared communication device. (b) Device mounted on a mobile robot.

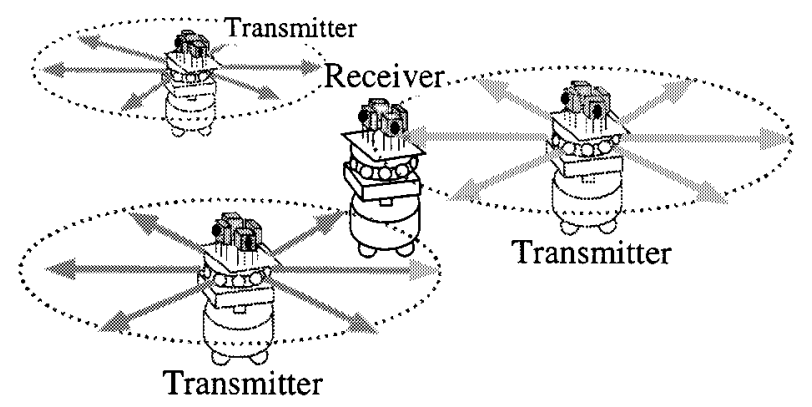

Figure 15. Experiment verificating optimal communication area (random search).

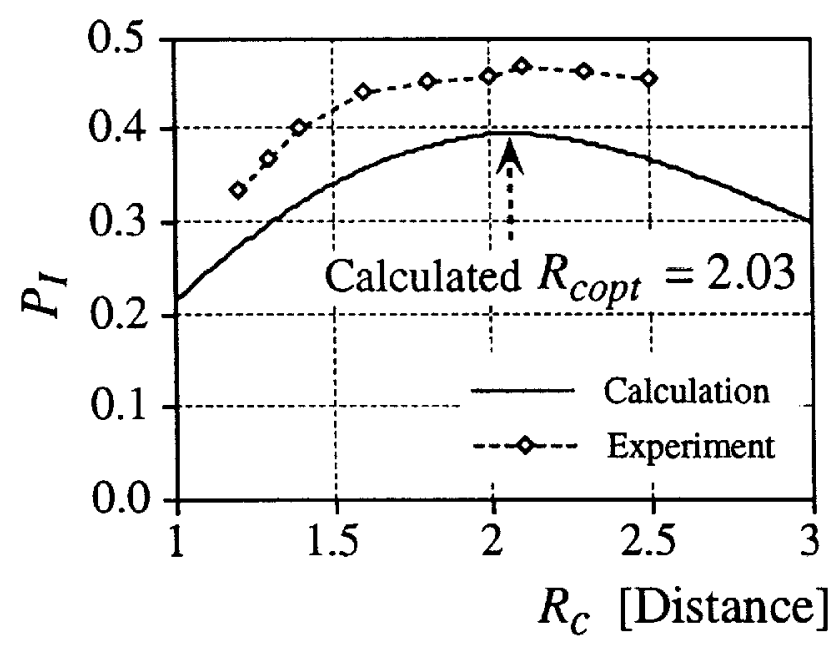

Figure 16. Experimental results of information transmission probability $P$.

Figure 16 shows the experimental result of information transmission probability $P$ compared to the theoretical value calculated, using the methodology described in Section 4.2.1. As seen in Figure $16, P$ takes the maximum in the experiment at the analytically derived optimal communication area $R_{\text {copt }}$. This demonstrates the effectiveness of the local communication area design.

We can also see that there is an offset between the experimental and analytical values. One reason for this is that the device does not realize interfering communication completely; i.e., when there are two robots on the same line from the receiver robot, the more distant robot is shaded by the nearer, so interference may not occur. This is to be studied in future work.

Although we only demonstrated the random search task, the effectiveness of the design for other tasks can be likewise verified.

\section{CONCLUSIONS}

We have demonstrated a design method for optimizing the local communication area for multiple mobile robots. Efficient cooperation requires that the communication system transmit task information to the appropriate robots in a minimum of time. For this purpose, the information transmissions are analyzed analytically to derive the optimal communication area.

We first modeled the spatial distributions for each task and derived the information transmission 
probability as an important measure for estimating the transmission time.

The next step of the analysis expressed the transmission time, using the information transmission probability in two cases: transmission to an arbitrary robot and to multiple robots. The optimal communication area was derived by minimizing the information transmission time.

We observed that the obtained optimal communication area increases as the robots' communication capacity increases, and is inversely proportional to the information output probability, which is determined from the cooperative tasks to be executed. As the design method determines the explicit relationship between the optimal area and the parameters characterizing the robots' capacity and tasks, it is able to adjust to configuration changes in the robotic system.

We verified the effectiveness of these analytical results by using computer simulations and experiments with multiple robots. The derived design method is useful for constructing an efficient local communication system for many robots.

\section{REFERENCES}

1. D. W. Gage, "Sensor abstractions of support manyrobot systems," Proc. SPIE Mobile Robots VII Conference, 1992, Vol. 1831, pp. 235-246.

2. L. Steels, "Cooperation between distributed agents through self-organization," in Decentralized AI, Y. Demazeau and J.-P. Muller, eds., North-Holland, Amsterdam, 1990, pp. 175-196.

3. M. Mataric, "Minimizing complexity in controlling a mobile robot population," Proc. IEEE Int. Conf. Rob. Autom., 1992, pp. 830-835.

4. R. Beckers, O. E. Holland, and J.-L Deneubourg, "From local actions to global tasks: Stigmergy and collective robotics," in Artificial Live IV, R. A. Brooks and P. Maes, eds., MIT Press, Cambridge, MA, 1994, pp. $181-189$.

5. D. Kurabayashi, J. Ota, T. Arai, and E. Yoshida, "Cooperative sweeping by multiple mobile robots," Proc. IEEE Int. Conf. Rob. Autom., 1996, pp. 1744-1749.

6. K. Sugihara and I. Suzuki, "Distributed algorithm for formation of geometric patterns with many mobile robots," J. Rob. Syst., 13, 127-139, 1996.

7. S. Ichikawa and F. Hara, "Experimental characteristics of multiple-robots behaviors in communication network expansion and object-fetching," in Distributed Autonomous Robotic Systems 2, H. Asama et al., eds. Springer, Berlin, 1996, pp. 224-234.

8. K. Singh and K. Fujimura, "Map making by cooperating mobile robots," Proc. IEEE Int. Conf. Rob. Autom., 2, 254-259, 1993.

9. G. Dudek, P. Freedman, and S. Hadjres, "Mapping in unknown graph-like worlds," J. Rob. Syst., 13, 539559, 1996.

10. A. Drogoul and J. Ferber, "From Tom Thumb to the dockers: Some experiments with foraging robots," Proc. 2nd Int. Conf. Simulation Adaptive Behavior, "From Animals to Animals 2," 1993, pp. 451-459.

11. M. Hashimoto, F. Oba, and T. Eguchi, "Dynamic control approach for motion coordination of multiple wheeled mobile robots transporting a single object," Proj. IEEE/RSJ Int. Conf. Intell. Rob. Syst. (IROS '93), 1993, pp. 1944-1951.

12. D. J. Stilwell and J. S. Bay, "Optimal control for cooperative mobile robots bearing a common load," Proc. IEEE Int. Conf. Rob. Autom. 1994, pp. 58-63.

13. N. Miyata, J. Ota, T. Arai, E. Yoshida, D. Kurabayashi, J. Sasaki, and Y. Aiyama, "Cooperative transport with regrasping of torque-limited mobile robots," Proc. IEEE/RSJ Int. Conf. Intell. Rob. Syst. (IROS '96), 1996, pp. 304-309.

14. S. Yuta and J. Iijima, "State information panel for inter-processor communication in an autonomous mobile robot-controller," Proc. IEEE Workshop Intell. Rob. Syst., 1990.

15. A. Asama, M. K. Habib, I. Endo, K. Ozaki, A. Matsumoto, and Y. Ishida, "Functional distribution among multiple mobile robots in an autonomous and decentralized robot system," Proc. Int. Conf. Rob. Autom., 1991, pp. 1921-1926.

16. F. R. Noreils, "An architecture for cooperative and autonomous mobile robots," Proc. IEEE Int. Conf. Rob. Autom., 1992, pp. 2703-2709.

17. J. Wang: “On Sign-board based inter-robot communication in distributed robotic systems," Proc. IEEE Int. Conf. Rob. Autom., 1994, 1045-1050.

18. T. Ueyama, T. Fukuda, and F. Arai, "Approach for self-organization-behavior, communication and organization for cellular robotic system," Proc. Int. Symp. Distributed Autonomous Rob. Syst. DARS '92, 1992, pp. 77-84.

19. S. Suzuki, H. Asama, A. Uegaki, S. Kotosaka, T. Fujita, A. Matsumoto, H. Kaetsu, and I. Endo, "An infrared sensory system with local communication for cooperative multiple mobile robots," Proc. IEEE/RSJ Int. Conf. Intell. Rob. Syst. (IROS '95), 1995, Vol. 1, pp. 220-225.

20. T. Arai, H. Kimura, J. Ota, and D. Kurabayashi, "Real-time measuring system of relative position on mobile robot system," Proc. Int. Symp. Indust. Rob., 1993, pp. 931-938.

21. R. C. Arkin, T. Balch, and E. Nitz, "Communication of behavioral state in multi-agent retrieval tasks," Proc. IEEE Int. Conf. Rob. Autom., 1993, pp. 588-594.

22. H. Takagi and L. Kleinrock, "Optimal transmission ranges for randomly distributed packet radio terminals," IEEE Trans. Commun. COM-32, 246-257, 1984.

23. T. Arai, E. Yoshida, and J. Ota, "Information diffusion by local communication of multiple mobile robots," Proc. IEEE Int. Conf. Syst. Man Cybernet., 1993, Vol. 4, pp. $535-540$.

24. R. M. Metcalfe and D. R. Boggs, "Ethernet: Distributed packet switching for local computer networks," Commun ACM, 19, 395-404, 1976. 